\title{
Efficacité des huiles essentielles de trois plantes aromatiques contre la mycoflore d'altération du niébé (Vigna unguiculata L., Walp) collecté dans les magasins de vente du Sud-Bénin
}

\author{
Brice Kpatinvoh, Euloge S. Adjou, Edwige Dahouenon-Ahoussi, T. R. Christian Konfo, Brice Atrevi, \\ Mohamed M. Soumanou, Dominique C. K. Sohounhloue \\ Laboratoire d'Etude et de Recherche en Chimie Appliquée, Ecole Polytechnique d'Abomey-Calavi, Université d'Abomey- \\ Calavi, 01 BP : 2009 Cotonou, (Bénin). \\ Adresse pour correspondance : edahoussi_95@yahoo.fr
}

Original submitted in on $13^{\text {th }}$ January 2017. Published online at www.m.elewa.orgon $31^{\text {st }}$ January 2017 http://dx.doi.org/10.4314/jab.v109i1.12

\section{RESUME}

Objectif : La présente étude vise à évaluer l'activité antimicrobienne des huiles essentielles extraites des feuilles fraîches de Cymbopogon citratus (L.), de Cymbopogon nardus (L.), et de Eucalyptus saligna (S.), sur les moisissures isolées des stocks de niébé de quelques magasins de vente du sud-Bénin.

Méthodologie et Résultats : Pour ce faire, un échantillonnage a été réalisé dans les grands magasins de stockage du niébé situés dans les marchés de Ouando (Porto-Novo) et de Dantokpa (Cotonou) suivi de la recherche et l'isolement de la mycoflore d'altération. Des tests antifongiques ont été réalisés avec les huiles essentielles de Cymbopogon citratus (L.), Cymbopogon nardus (L.), et de Eucalyptus saligna (S.), afin d'évaluer leur potentiel antifongique. Des résultats obtenus, il ressort que les souches fongiques isolées des échantillons de niébé sont essentiellement Aspergillus flavus (L.), Aspergillus tamarii (K.) et Penicillium roqueforti (T.). Les tests antifongiques ont permis de démontrer l'activité des huiles essentielles testées contre la flore fongique contaminant le niébé, avec des concentrations minimales d'inhibition (CMI) dépendantes du type fongique et de la dose d'huile essentielle testée. Les fortes activités antifongiques sont obtenues avec les huiles essentielles de Cymbopogon citratus (L.) et de Cymbopogon nardus (L.) avec des CMI comprises entre de 0,05 et $1 \mu \mathrm{l} / \mathrm{ml}$.

Conclusion: Le potentiel antifongique de ces plantes offre donc une approche novatrice dans la gestion intégrée des stocks de niébé au Sud du Bénin.

Mots clés : niébé, moisissures, huiles essentielles, activité antifongique.

\section{ABSTRACT}

Objective: The aim of this study was to investigate the antimicrobial activity of essential oils extracted from fresh leaves of Cymbopogon citratus (L.), Cymbopogon nardus (L.), and Eucalyptus saligna (S.), on isolated fungi of cowpea collected from a few retail store in southern Benin.

Methodology and Results: Sampling was carried out in a few large cowpea retail store located in the markets of Dantokpa (Cotonou) and Ouando (Porto-Novo), followed by research and isolation of the alteration mycoflora. Antifungal tests were carried out with various essential oils in other to assess their antimicrobial potential. 
Results obtained, indicated that the microbial strains isolated from the cowpea samples are essentially Aspergillus flavus (L.), Aspergillus tamarii (K.) and Penicillium roqueforti (T.). Antimicrobial tests have demonstrated that essential oils have high antimicrobial activities against fungal flora contaminating cowpea with minimal inhibition concentrations depend on fungi and the dose of essential oil tested. The highest antifungal activities were obtained with essential oils from Cymbopogon citratus (L.) and Cymbopogon nardus (L.) with MIC ranged from 0.05 to $1 \mu \mathrm{l} / \mathrm{ml}$.

Conclusion: The antifungal potential of these plants therefore offers an innovative approach in the management of cowpea stocks in southern Benin.

Keywords: cowpea, fungi, essential oils, antifungal activity, Benin.

\section{INTRODUCTION}

Les problèmes rencontrés en Afrique par les producteurs pendant la phase post-récolte des produits agricoles ont été longtemps négligés, confondus à ceux liés à la production. Pendant ce temps, les pertes post-récoltes enregistrées sont de plus en plus croissantes, car les technologies traditionnelles de stockage et de transformation mises en œuvre sont généralement inadéquates avec des risques d'infestation des produits stockés par des moisissures toxinogènes (Fandohan et al., 2003). Le niébé (Vigna unguiculata L., Walp) constitue la légumineuse à graine la plus importante en Afrique tropicale (Zannou, 1995). Nonobstant son importance, les pertes causées par les insectes et les moisissures sont les plus importantes, en raison du climat tropical et inter-tropical très favorable à leur développement et du type de stockage, peu propice à la lutte contre les insectes ravageurs des stocks (de Groot, 2004). Malgré l'existence d'une gamme de technologies visant la réduction des pertes postrécolte, plusieurs contraintes persistent encore au niveau du processus stockage/conservation, ainsi que la transformation du niébé. Ces contraintes sont beaucoup plus accentuées au niveau de nos marchés où la méthode de conservation du niébé favorise la prolifération des moisissures et des déprédateurs dans les produits stockés. La lutte chimique a toujours été la principale mesure pour réduire l'incidence des contaminations post-récoltes dans les produits alimentaires. Cependant, l'application à des concentrations élevées de ces produits chimiques synthétiques dans une

\section{MATERIEL ET METHODES}

Échantillonnage du niébé : Les graines de niébé utilisées dans cette étude sont collectées dans les grands perspective de contrôle post-récolte des denrées alimentaires augmente le risque de résidus toxiques dans les produits alimentaires (Hammer et al., 1999). En raison de la sensibilité croissante des consommateurs à cette pollution résiduelle et des effets toxiques de nombreux fongicides de synthèse, l'importance de l'utilisation de produits alternatifs naturels devient nécessaire (Bankole, 2004). De même, la restriction imposée par l'industrie alimentaire et les organismes de réglementation sur I'utilisation de certains additifs alimentaires synthétiques ont conduit à un regain d'intérêt dans la recherche d'alternatives, comme des composés naturels, en particulier ceux d'origine végétale (Hammer et al., 1999). Plusieurs études ont montré que les extraits de plantes possèdent de nombreuses propriétés dont l'une des plus importantes est l'activité antimicrobienne (Yehouenou et al., 2010; Soumanou et Adjou 2016). L'utilisation des huiles essentielles en tant qu'agents antimicrobiens présente deux avantages principaux : le premier est leur origine naturelle qui signifie plus de sécurité pour la population et l'environnement et le second est qu'elles sont considérés à faible risque de développement de résistance par des microorganismes pathogènes (Tatsadjieu et al., 2010). Ainsi, la présente étude vise à évaluer l'activité antifongique des huiles essentielles de de Cymbopogon citratus (L.), de Cymbopogon nardus (L.) et de Eucalyptus saligna (S.) sur les moisissures isolées des stocks de niébé en conservation dans quelques grands magasins de vente du Sud-Bénin.

magasins de stockage du niébé situés dans les marchés de Ouando (Porto-Novo) et de Dantokpa (Cotonou). Le 
choix de ces magasins de stockage est basé sur le fait qu'ils constituent des zones d'approvisionnement pour les vendeurs grossistes qui se chargent ensuite de les livrer aux vendeurs détaillants.

Détermination de la teneur en eau des graines de niébé collectée : La teneur en eau des échantillons de niébé collectés, a été déterminée par étuvage direct de $5 \mathrm{~g}$ d'échantillon à $105^{\circ} \mathrm{C}$ pendant 24 heures, de, selon la méthode décrite par De Knegt and Brink (1998).

Évaluation de la pression parasitaire fongique : Afin d'évaluer l'étendue de la contamination par les moisissures dans les différents échantillons d'arachide collectés, le taux de contamination fongique des échantillons a été investigué. La technique de l'ensemencement direct (Direct Plating) décrit par Pitt et al. (1994) a été utilisée. C'est l'une des méthodes appropriées pour détecter, évaluer le taux de contamination des échantillons et isoler des mycètes des denrées alimentaires (N'Guyen, 2007). Par échantillon, cent (100) graines de niébé ont été prélevées puis désinfectées en surface grâce à une solution chlorée $(0,4 \%)$ pendant une minute à température ambiante. Elles sont ensuite placées sur le milieu de culture Yeast Extract Sucrose Agar (YES) préalablement coulé dans des boîtes de Pétri de $9 \mathrm{~cm}$ de diamètre. Les boites de Pétri ainsi ensemencées sont ensuite incubées à $25^{\circ} \mathrm{C}$ pendant cinq jours. Cette méthode permet d'isoler prioritairement la flore fongique se trouvant à lintérieur des graines. La méthode d'ensemencement par dilution a été également utilisée dans le but de rechercher la flore fongique se trouvant à la surface des graines. La technique utilisée est celle décrite par Nguyen (2007). Vingt-cinq (25) grammes de chaque échantillon ont été prélevés et additionnés à $225 \mathrm{ml}$ d'eau peptonée tamponée. Le mélange a été agité pendant 15 minutes. Ensuite, 0,1 millilitre de cette suspension a été étalé sur le milieu de culture YES. Les boites de Pétri ont été incubés à $25^{\circ} \mathrm{C}$ pendant 5 jours. Le taux de contamination (TC) par échantillon a été calculé selon la formule suivante : TC= (NI / NT) x 100 où $\mathrm{N}_{\mathrm{T}}$ représente le nombre de graines présentant un développement de moisissures et $\mathrm{NI}$ le nombre total de graines (Adjou et al., 2013).

Isolement et identification des mycètes : Les moisissures qui se sont développées sur les différents milieux de culture ont été purifiées par repiquages répétés. Les cultures pures de moisissures ont été examinées macroscopiquement et microscopiquement, et leur identification a été réalisée à l'aide du schéma taxonomique basé sur l'aspect des vésicules, des métula, des phialides et l'onctogénicité des spores en utilisant la méthode décrite par Singh et al. (1991), Filtenborg et al.
(1995) et Tabuc (2007). La fréquence d'apparition de chaque moisissure a été ensuite calculée.

Collecte du matériel végétal et extraction des huiles essentielles : Le matériel végétal utilisé est constitué des feuilles fraiches de Cymbopogon citratus (L.), de Cymbopogon nardus (L.), et de Eucalyptus saligna (S.), collectées à Abomey-Calavi (Sud du Bénin) et identifiées par l'Herbier National. L'extraction des huiles essentielles a été effectuée, par hydrodistillation grâce à un appareil de type Clevenger. L'huile essentielle séparée de la phase aqueuse a été séchée sur le sulfate de sodium (Na2SO4) et conservé à $5^{\circ} \mathrm{C}$ à l'obscurité. Le rendement d'extraction $(R)$ est exprimé par rapport au matériel végétal frais et son calcul s'est fait de la manière suivante

$\mathrm{R} \%=(\mathrm{Mh} / \mathrm{Ms}) \times 100$ où Mh est la masse d'huile essentielle obtenue et Ms représente la masse de matière végétale utilisée.

Tests antifongiques: L'activité antimicrobienne des huiles essentielles a été évaluée in vitro et a pour but de déterminer la concentration minimale inhibitrice (CMI) et la concentration minimale fongicide (CMF) de ces huiles essentielles. Ces tests ont été effectués en milieu solide sur les différents types de moisissures isolées des échantillons de niébé collectés. La détermination de la $\mathrm{CMI}$ a été réalisée par la méthode décrite par deBillerbeck et al. (2001) et Adjou et al. (2012). Dans des boites de petri de $9 \mathrm{~cm}$ de diamètre et contenant chacun $20 \mathrm{ml}$ du milieu de culture Sabouraud au Chloramphénicol, stérilisé pendant $15 \mathrm{~min}$ à $121^{\circ} \mathrm{C}$ et refroidi à $45^{\circ} \mathrm{C}$, on ajoute aseptiquement des quantités décroissantes d'huiles essentielles. Des disques mycéliens de $6 \mathrm{~mm}$ de la souche fongique à tester sont déposés au centre de chaque boite de pétri. Des témoins sans extraits ont été réalisés. L'incubation est réalisée à $28^{\circ} \mathrm{C}$ pendant 5 jours. Les diamètres de la croissance mycélienne sont mesurés et comparés à celui de témoin. La CMI correspond à la plus faible concentration à partir de laquelle aucune croissance fongique n'est observée. Aux concentrations expérimentales où aucune croissance ni germination n'est observée, nous avons testé l'activité fongicide ou fongistatique. Ce test consiste à prélever le disque mycélien qui n'a pas poussé en fin d'incubation de la boite de Pétri et à le réintroduire dans un milieu de culture neuf sans huile essentielle. Dans le cas où la croissance mycélienne est toujours inhibée, on parle d'activité fongicide de l'huile essentielle et dans le cas contraire, il s'agit de l'activité fongistatique (Yehouenou et al., 2012).

Analyses statistiques : Les résultats ont été analysés par la méthode de variance (ANOVA) à l'aide du logiciel 
STATISTICA (Stat., Soft, Inc, 1995). La comparaison des moyennes est effectuée par le test de la plus petite différence significative LSD (Least Significant Difference).

\section{RESULTATS ET DISCUSSIONS}

Rendement d'extraction: Les rendements obtenus après extraction des huiles essentielles des trois plantes investiguées sont présentés dans le Tableau 1. L'analyse de ce tableau montre que les rendements en huile essentielle varient d'une espèce végétale à une autre. Ces rendements sont compris entre $1,72 \%$ et $6,88 \%$. Le rendement en huile essentielle de Cymbopogon citratus (L.) est proche à celui obtenu par Ayédoun et al.,(1997) Nonviho (2010) et Konfo et al. (2012) qui ont étudié les huiles essentielles extraites des feuilles récoltées au
Cette méthode d'analyse consiste à chercher les moyennes qui diffèrent significativement les unes des autres. Les différences sont significatives lorsque $P<0.05$.

Tableau 1: Rendement d'extraction des huiles essentielles

\begin{tabular}{|l|l|c|}
\hline \multicolumn{1}{|c|}{ Espèces végétales } & Familles botaniques & $\begin{array}{c}\text { Rendement en huile essentielle } \\
(\mathbf{\%})\end{array}$ \\
\hline Cymbopogon nardus & Poaceae & 6,88 \\
\hline Cymbopogon citratus & Poaceae & 4,31 \\
\hline Eucalyptus saligna & Myrtaceae & 1,72 \\
\hline
\end{tabular}

Humidité des graines de niébé collectées : Le tableau 2 présente les résultats de l'évaluation du taux d'humidité des échantillons de graine de niébé collectés dans les marché de Marché Dantokpa (Cotonou) et de Marché de Ouando (Porto-Novo). De l'analyse de ces résultats, il ressort que le taux d'humidité des graines de niébé collectées varie de $1,66 \pm 0,04 \%$ à $1,89 \pm 0,01 \%$. Les taux d'humidité élevés observés dans les échantillons collectés dans le marché de Dantokpa (Cotonou) pourraient être liés à la méthode de conservation des graines dans les magasins de stockage, mais aussi à
Bénin. Ce rendement est largement supérieur à celui obtenu par François et al. (2009) et Kanko (2010) qui sont respectivement 0,67 et $0,7 \%$ sur la même espèce végétale. Par contre le rendement en huile essentielle de Cymbopogon nardus est proche à celui obtenu par Reis et al. (2006) et supérieur à celui obtenu par Silva et al. (2011). Ces différences observées au niveau des rendements pourraient être liées à la zone de collecte, la nature du sol, et le stade de développement de la plante.

Tableau 2: Taux d'humidité des échantillons de niébé collectés

\begin{tabular}{|l|c|c|}
\hline \multicolumn{1}{|c|}{ Lieux de collecte } & Zone agroécologique & Humidité (\%) \\
\hline Marché Dantokpa (Cotonou) & $\begin{array}{c}\text { Zone des pêcheries et des cultures } \\
\text { maraîchères }\end{array}$ & $1,89 \pm 0,01 \mathrm{a}$ \\
\hline Marché de Ouando (Porto-Novo) & Zone des terres de barre & $1,66 \pm 0,04 \mathrm{~b}$ \\
\hline
\end{tabular}

Les valeurs sont des moyennes $(n=3)$. Les valeurs portant la même lettre dans la même colonne ne sont pas significativement différentes $(p<0,05)$

Flore fongique des échantillons de niébé collectés : Les figures 1, 2 et 3 présentent l'aspect macroscopique et microscopique des différentes espèces de moisissures isolées des échantillons de niébé collectés. D'après les observations macroscopique et microscopique, deux genres de moisissures répartis dans trois espèces sont identifiées. Il s'agit de Aspergillus flavus (L.), Aspergillus l'humidité relative de l'air dans cette zone agroécologique. En effet, la zone des pêcheries et des cultures maraîchères (Zone agroécologique VIII), située au sud du Bénin possède une humidité relative très élevée à cause de l'alizée maritime qui souffle au-dessus de la surface de l'Océan Atlantique (Adjou et Soumanou, 2013). Ces vents recueillent l'eau qui s'évapore de la surface des mers et jouent ainsi un rôle fondamental dans le soulèvement à très haute altitude de l'air équatorial par convection humide après qu'ils aient atteint la zone de convergence intertropicale. 
et Pfohl-Leszkowicz, 2002). Ces moisissures peuvent se développer dans les aliments pauvres en eau au cours du stockage et les produits dont l'activité hydrique a été réduite. Selon Riba et al. (2005), le manque de ventilation couplé à une température élevée, favorise la croissance des champignons, comme Aspergillus et Penicillium. Houssou et al. (2009) ont isolé et identifié Aspergillus flavus dans les échantillons de niébé récoltés au Bénin. Cette moisissure productrice potentielle d'aflatoxine contamine les graines de niébé. Ainsi, la durée de stockage est un facteur qui influence l'incidence de ces espèces de moisissures sur les graines. Les facteurs zone de collecte et type de variété sont sans effet significatif sur cette incidence (Houssou et al., 2009).

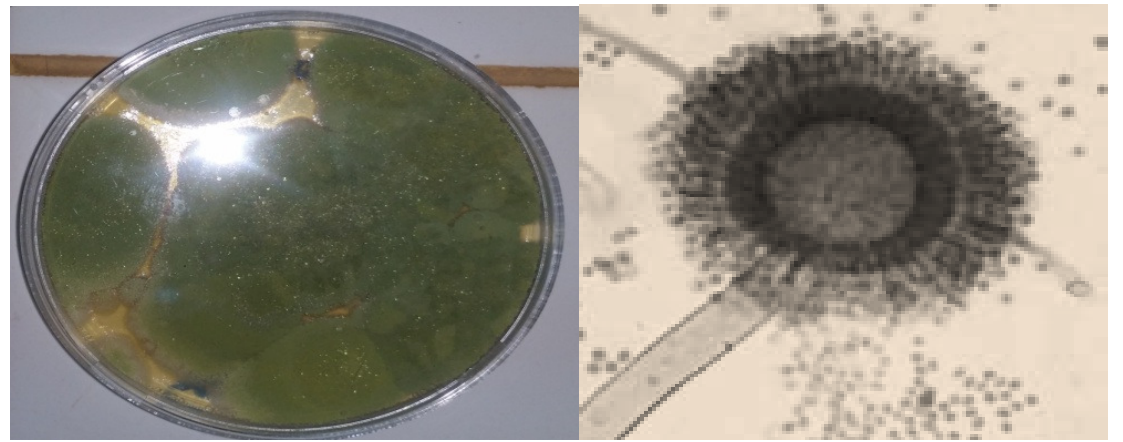

Figure 1 : Aspects macroscopique et microscopique des isolats d'Aspergillus flavus (L.) qui contaminent le niébé en post-récolte

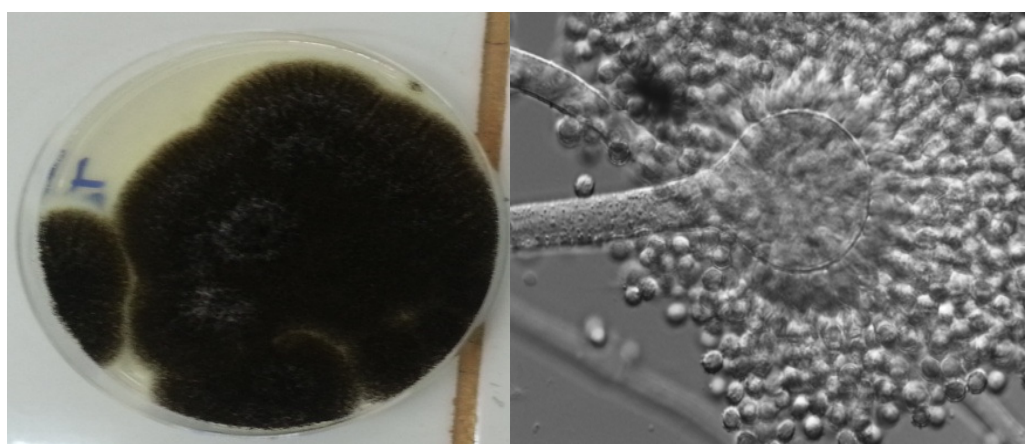

Figure 2 : Aspects macroscopique et microscopique des isolats d'Aspergillus tamarii (K.) qui contaminent le niébé en post-récolte

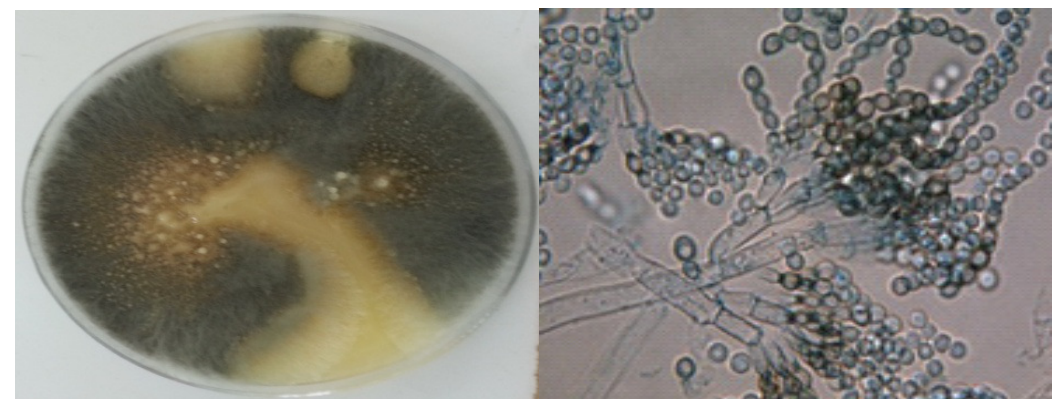

Figure 3 : Aspects macroscopique et microscopique des isolats de Penicillium roqueforti (T.) qui contaminent le niébé en post-récolte

Tests antifongiques : Les résultats de la détermination des concentrations minimales d'inhibition (CMI) et concentrations minimales fongicides (CMF) des différentes huiles essentielles testées sur les souches fongiques isolées des échantillons de niébé sont présentés dans le Tableau 3. De l'analyse de ces 
résultats, il ressort que les huiles essentielles de Cymbopogon citratus (L.), de Cymbopogon nardus (L.), sont plus actives sur les souches microbiennes testées, que l'huile essentielle de Eucalyptus saligna (S.). Cette forte activité des huiles essentielles de Cymbopogon citratus (L.), de Cymbopogon nardus (L.), pourrait être en dépendre de leur composition chimique. En effet, l'huile essentielle de Cymbopogon citratus (L.), collectée au Sud du Bénin est majoritairement composée de géranial $(41,3 \%)$, de néral $(33 \%)$, de myrcène $(10,4 \%)$, de acétate de géranyle (2,4\%) (Konfo, 2012), et celle de Cymbopogon nardus (L.) est majoritairement composée de citronellal $(30,54 \%)$, de géraniol $(23,93 \%)$ et de citronellol (7,65\%) (Koba et al., 2009). Ces deux huiles essentielles sont majoritairement composées de monoterpènes oxygénés $(87,0 \%$ - 74,16\%). Ainsi, l'activité antifongique des huiles essentielles de
Cymbopogon citratus (L.) et de Cymbopogon nardus (L.) serait due à l'action des monoterpènes oxygénés. En effet, les travaux de Yoshimura et al., (2010) ont montré que les huiles essentielles riches en monoterpènes oxygénés possèdent des effets délétères sur les membranes mitochondriales des moisissures, ce qui provoque une inhibition du métabolisme énergétique mitochondrial et entraine des perturbations des processus physiologiques et biochimiques de la cellule. L'activité antifongique modérée de l'huile essentielle de $E$. saligna serait due à sa composition chimique qui diffère de celles des huiles essentielles de Cymbopogon citratus (L.) et de Cymbopogon nardus (L.). De même, l'activité antimicrobienne des huiles essentielles est non seulement le résultat de ses composés majoritaires mais aussi l'effet de synergie entre les composés minoritaires (Soumanou and Adjou 2016).

Tableau 3 : Activités antifongiques des huiles essentielles testées sur les souches fongiques isolées des échantillons de niébé collectés

\begin{tabular}{|c|c|c|c|c|}
\hline \multirow{2}{*}{$\begin{array}{c}\text { Souches } \\
\text { fongiques }\end{array}$} & \multirow{2}{*}{$\begin{array}{l}\text { Concentrations } \\
\text { Minimales }\end{array}$} & \multicolumn{3}{|c|}{ Huiles essentielles testées } \\
\hline & & $\begin{array}{l}\text { Cymbopogon } \\
\text { citratus }\end{array}$ & Cymbopogon nardus & Eucalyptus saligna \\
\hline \multirow[t]{2}{*}{ Aspergillus flavus } & $\mathrm{CMI}(\mu \mathrm{l} / \mathrm{ml})$ & 0,05 & 1 & 15 \\
\hline & $\operatorname{CMF}(\mu l / m l)$ & 1 & 2 & 20 \\
\hline \multirow[t]{2}{*}{ Aspergillus tamarii } & $\mathrm{CMI}(\mu \mathrm{l} / \mathrm{ml})$ & 0,05 & 0,2 & 15 \\
\hline & $\mathrm{CMF}(\mu l / m l)$ & 1 & 1 & 20 \\
\hline \multirow{2}{*}{$\begin{array}{l}\text { Penicillium } \\
\text { roqueforti }\end{array}$} & CMI $(\mu l / m l)$ & 0,05 & 0,05 & 10 \\
\hline & $\mathrm{CMF}(\mu l / m l)$ & 1 & 1 & 15 \\
\hline
\end{tabular}

\section{CONCLUSION}

La présente étude réalisée sur les échantillons de niébé en post-récolte a montré que ces échantillons sont contaminées par les moisissures, notamment Aspergillus flavus, Aspergillus tamarii et Penicillium roqueforti Les tests antifongiques réalisés sur les souches microbiennes isolées, ont montré que les huiles essentielles de Cymbopogon citratus (L.) et Cymbopogon nardus (L.)

\section{REFERENCES}

Adjou ES, Soumanou MM, 2013. Efficacité des extraits de plantes dans la lutte contre les moisissures toxinogènes isolées de l'arachide en postrécolte au Bénin. Journal of Applied Biosciences, 70 : 5555- 5566.

Adjou ES, Kouton S, Dahouenon-Ahoussi E, Soumanou MM, Sohounhloue DCK, 2013. Effect of Essential Oil from fresh Leaves of Ocimum possèdent des propriétés antifongiques très prononcées sur les trois espèces de moisissures isolées. Par contre, I'huile essentielle de Eucalyptus saligna (S.) s'est révélée moins active sur les souches microbiennes testées. Ces huiles essentielles peuvent donc constituer une alternative crédible dans la lutte contre les moisissures contaminant le niébé en post-récolte au Bénin.

gratissimum L. on Mycoflora during Storage of Peanut in Benin. Mycotoxin Research, 29:2938.

Ayedou MA, Sohounhloué DK, Moudachirou M, Lamaty G, 1997. Composition chimique des huiles essentielles de deux espèces de Cymbopogon du Bénin exploitables industriellement. Bioressources Energie, $8: 4-5$. 
Bankole S, Mabekoje 00, 2004. Mycoflora and occurrence of aflatoxin B1 in dried yam chips from markets in Ogun and Oyo States, Nigeria. Mycopathologia, 157: 111-115.

Castegnaro M., Pfohl-Leszkowicz A., (2002). Les mycotoxines: contaminants omniprésents dans l'alimentation animale et humaine. Moll \& Moll (eds) Lavoisier

De Billerck G, 2001. Activité fongique de l'huile essentielle de Cymbopogon nardus sur Aspegillus niger. Evaluation d'un bioréacteur pour l'étude de l'effet inhibiteur des substances volatiles en phase vapeur. Thèse de Doctorat, Faculté des sciences pharmaceutiques, Institut National Polytechnique de Toulouse. 239p.

de Groot I, 2004. Agrodok 18 Protection des céréales et des légumineuses stockées $2^{\text {ème }} \mathrm{Ed} \quad, 22 \mathrm{p}$.

De Knegt R.J. and Brink Hvd, 1998. Improvement of the drying oven method for the determination of the Moisture Content of Milk Powder. Int. Dairy Journal, 8:733-738.

Fandohan $P$, Zoumenou D, Hounhouigan DJ, Marasas WFQ, Wingfield MJ, Hell K, 2005. Fate of aflatoxins and fuminosins during the processing of into food products in Benin. Int. J. Food Microbiol. 98:249-259.

Filtenborg O, Frisvad JC, Thrane U, 1995. Moulds in food spoilage. International Journal of Food Microbiology, 33:85-102.

Hammer KA, Carson CF, Ridley CV, 1999. Antimicrobial activity of essential oils and other plants extract. Journal of Applied Microbiology, 86:985-990.

Houssou PA, Ahohuendo BC, Fandohan P, Kpodo K, Hounhouigan DJ, Jakobsen M, 2009. Natural infection of cowpea (Vigna unguiculata (L.) Walp.) by toxigenic fungi and mycotoxin contamination in Benin, West Africa. Journal of Stored Products Research, 45: 40-44.

Kanko C, 2010. Contribution à l'étude phytochimique de plantes médicales et aromatiques de Côte d'Ivoire. Activité analgésique et antiinflammatoire de stérols isolés de l'écorce de Parkia biglobosa. Thèse de doctorat, Université de Cocody-Abidjan., Abidjan, 224p.

Koba K, Poutouli PW, Raynaud C, Chaumont J.P, and Sanda K. 2009. Chemical composition and antimicrobial properties of different basil essential oils chemotypes from Togo. Bangladesh J Pharmacal, 4: 1-8.

Konfo C, Ahoussi-Dahouenon E, Sessou P, Yehouenou B, Djenontin S, de Souza C, Sohounhloue D,
2012. Stabilization of Local Drink "Tchakpalo" produced in Benin by addition of Essential Oil Extracted from Fresh leaves of Cymbopogon citratus International Research Journal of Biological Sciences, 1(8): 40-49.

N'guyen M, 2007. Identification des espèces de moisissures potentiellement productrices de mycotoxines dans le riz commercialisé dans cinq provinces de la région centrale du Vietman Étude des conditions pouvant induire la production de mycotoxines. Institut National Polytechnique de Toulouse (INPT). Thèse de doctorat. $147 \mathrm{p}$.

Nonviho G, Wotto VD, Noudogbessi J-P, Avlessi F, Akogbeto M, Sohounhloué DC, 2010. Insecticidal activities of essential oils extracted from three species of poaceae on Anopheles gambiae, major vector of malaria 4: 411-420.

Pitt $\mathrm{Jl}$ et Miscamble BF, 1995. Water relations of Aspergillus flavus and closely related species. Journal of Food Protection, 58: 86-90.

Pitt JI, Hocking AD, Bhudhasamai K, Miscamble BF, Wheeler KA, Tanboon EKP, 1994. The normal mycoflora of commodities from Thailand. 2: Beans, rice, small grains and other commodities. International Journal of Food Microbiology, 23: 35- 53.

Riba A., Sabaou N., Mathieu F. et Lebrihi A., 2005. Premières investigations sur les champignons producteurs d'Ochratoxine $A$ dans la filière céréale en Algérie. Symposium Euro-Maghrébin sur les contaminants biologiques, chimiques et la sécurité alimentaire, Fès.

Silva CF, Moura FC, Mendes MF, Pessoa FLP, 2011. Extraction of citronella (Cymbopogon nardus) essential oil using supercritical $\mathrm{CO}_{2}$ : experimental data and mathematical modeling. Brazilian Journal of Chemical Engineering, 28 : 343-350.

Singh K, Frisvad JC, Thrane U, Mathu SB, 1991. An illustrated manual on identification of some seed borne Aspergilli, Fusaria, Penicillia and their mycotoxins. Danish Government, Institute of seed pathology for developing countries, Hellerup, Denmark.

Soumanou MM, Adjou ES, 2016. Sweet Fennel (Ocimum gratissimum) Oils. In:Preedy, V.R. (Ed.), Essential Oils in Food Preservation, Flavor and Safety. Academic Press, 765-773.

Tabuc C, 2007. Flore fongique de différents substrats et conditions optimales de production des mycotoxines. Thèse de doctorat. Institut National 
Polytechnique de Toulouse et Université de Bucarest.

Tatsadjieu N, Jazet M, Ngassoum MB, Etoa X, Mbofung MF, 2010. Investigations on the essential oil of Lippia rugosa from Cameroon for its potential use as antifungal agent against Aspergillus flavus Link ex. Fries. Food Control, 5:161-166.

Yèhouénou $B$, Noudogbessi J-P, Sessou P, Avlessi $F$, Sohounhloué Dominique, 2010. Étude chimique et activités antimicrobiennes d'extraits volatils des feuilles et fruits de Xylopia aethiopica (Dunal) A. Rich. Contre les pathogènes des denrées alimentaires. Journal de la Société Ouest-Africaine de Chimie, $029: 19$ - 27.

Yehouenou B, Ahoussi E, Sessou P, Alitonou GA, Toukourou F, Sohounhloue CKD, 2012. Chemical composition and antimicrobial activities of essential oils (EO) extracted from leaves of Lippia rugosa A. Chev against foods pathogenic and adulterated microorganisms. African Journal of Microbiological Research, 6(26):5496-5505.

Yoshimura H, Sawai Y, Tamotsu S, Sakai A, 2010. 1,8cineole inhibits both proliferation and elongation of BY-2 cultured tobacco cells, J Chem Ecol, 37(3):320-328.

Zannou ET, 1995. Observation des pontes de bruches et de leurs hyménoptères parasitoïdes oophages dans un système de culture du niébé, Vigna unguiculata (L) Walp, au Sud Bénin. Mémoire de DEA, Université du Bénin, Lomé, (Togo) 39 p. 